\title{
Hearing is believing: Birds learn fear
}

\author{
Christopher B. Sturdy ${ }^{1,2}$ • Darren S. Proppe ${ }^{3}$
}

Published online: 17 December 2015

(C) Psychonomic Society, Inc. 2015

Summary Although it is known that animals attend to the vocalizations of others (referred to as eavesdropping), what has been missing, or at least left experimentally unproven, until now is whether animals can learn new associations between a signal and a threat. Here Magrath and colleagues (Current Biology, 25(15), 2047-2050, 2015) have for the first time conducted a field experiment that demonstrates just this: superb fairy-wrens learned to associate a novel vocalization with a predator.

\section{Introduction}

Eavesdropping is generally considered impolite and frowned upon when we humans engage in such activity. For other animals, eavesdropping can be an effective way to gain information that can improve, extend, or in cases like the experiment conducted by Magrath and colleagues (2015). save their lives. Evidence that animals attend to the communication of others is substantial and exists both within and across species boundaries (e.g., Dabelsteen, 2005)'. One main area of investigation regarding heterospecific eavesdropping has centered around deciphering alarm vocalizations of other species, that is, vocalizations that signal the presence of a predator and would lead animals to take evasive actions such as flight, freezing, or mobbing behaviors, otherwise aimed at avoiding predation (Magrath et al., 2014).

What is less clear from previous research is whether animals that listen in on other animals' vocalizations are learning anything new or are simply attending to other species' vocalizations because they are acoustically similar to their own alarm calls. While such behavior could lead to similar outcomes (i.e., extending

Christopher B. Sturdy

csturdy@ualberta.ca

1 Department of Psychology, University of Alberta, Edmonton, AB, Canada

2 Neuroscience and Mental Health Institute, University of Alberta, Edmonton, AB, Canada

3 Department of Biology, Calvin College, Grand Rapids, MI, USA one's life), the mechanisms underlying such behavior would necessarily not be as flexible as one that involved learned associations between signals and predators. In this recent set of experiments, Magrath and colleagues set out to fill this gap in our understanding of eavesdropping behavior. To address this problem, the group conducted a field experiment with superb fairy-wrens, a small Australian songbird, as subjects. In their clever and wellcontrolled design, they exposed wrens to synthetic and unfamiliar bird calls, presented in two different ways, either paired with a model of a predator or not. Following training, birds were tested to determine their responses. As one might expect, the birds that were presented the stimuli in an unpaired manner did not exhibit any anti-predator behavior, demonstrating that mere exposure to the stimuli in the absence of training did not lead to avoidance behavior. In stark contrast, birds in the experimental group who were trained to associate previously unfamiliar stimuli with the appearance of a predator model resoundingly, and in almost all cases, fled the scene to the cover of nearby foliage. Impressively, these changes in the behavior of experimental birds persisted when tested 1 and 2 days after training.

\section{Implication 1: Learning principles act in nature}

Determining which mechanisms underlie behavior in nature is highly complex, largely due to the number and variability of potentially contributing factors. Nonetheless, Magrath et al. have convincingly provided proof of principle that indeed, at least for superb fairy-wrens, birds can learn to associate novel acoustic signals that bear no resemblance to their own alarm calls, with a predator. Whether this phenomenon is common remains to be tested in other bird species and different taxa; the result is nonetheless impressive and not likely a single case. Such hopefulness is warranted in part because of the attention to detail and experimental design paid by this group. With this solid design, they combined their knowledge of their study species and in so doing eliminated other possible pitfalls, or at least tested for and ruled them out. To wit: they used two stimuli that were both unfamiliar to their study species and acoustically dissimilar, thereby controlling for both factors; they then tested birds' 
reactions in a baseline condition, to ensure that the stimuli on their own, or the birds in the absence of training, would not flee irrespective of what stimulus was presented. Using this systematic approach allowed the researchers to conclude that birds can learn to associate novel signals with predators, and by extension, that these learning principles can operate in nature and lead to adaptive changes in behavior.

\section{Implication 2: Learning principles can be used for conservation}

An obvious implication and possible extension of this work was noted by Magrath and colleagues but deserves mentioning here again, namely that similar learning principles can be employed in a conservation setting. As they notably point out, captive breeding programs for endangered species could adopt a similar protocol to enhance survival of naïve animals prior to their release into the wild. The manner in which Magrath and colleagues trained their birds was simple and effective. That they could do so in the wild and have lasting, significant results should bode very well for captive breeding programs that would have, by definition, much greater control over subjects. This control, while an experimental benefit on the one hand, can also result in a potential hazard that should not be overlooked, and that is the role of contextual learning. Just as treatments of human phobias need to generalize outside of a therapist's office to be of benefit to the client, so too would antipredator training conducted in a zoo or sanctuary. This could potentially be accomplished by conducting "reminder" sessions upon release. In spite of this potential wrinkle, there is reason to hope that these techniques can be widely adopted and increase the survivability of captivebred, or rehabilitated, animals when (re)released into the wild.

\section{Implication 3: Learning and behavioral ecology researchers have much to gain from one another}

What should be obvious from this paper is the opportunity that it presents, not only for the many follow-up studies that will surely follow this initial result, but more broadly, the potential for gaining significant insight into the natural history of animals when integrative research is conducted at a high level. This is not at all to disparage pure practitioners of either discipline represented here, namely animal learning or behavioral ecology. Rather, if anything, what this study shows us is how pure research principles from each discipline, important in its own right, can and should be fruitfully combined. By drawing on the best of both research worlds, Magrath and colleagues were able to produce a durable and meaningful result that might not have been possible had they not conducted their research with such interdisciplinary rigor. Within their own fields, it may seem that neither group has much in common with the other: learning researchers often use a small number of species (rats and pigeons) tested in unnatural experimental conditions, while behavioral ecologists use the seemingly endless variety provided by nature, in nature. That said, the principles studied in the laboratory are general processes, applicable to much of nature, and the experimental design and control procedures, while seemingly restrictive and pedantic, are actually freeing and allow for more firm conclusions. Similarly, paying close attention to how animals actually behave in nature as well as addressing issues important to their survival and fitness, are questions, pardon the turn of phrase, of ultimate importance, irrespective of your particular research area.

\section{Implication 4: Fundamental research is essential for building a solid foundation upon which hypotheses can be generated and tested}

Finally, we would like to point out an even more overarching implication of this particular study that extends beyond the particular conclusions, and instead speaks to the value of fundamental research in behavioral biology and psychology more generally. This study would not have been possible without the myriad of other studies that came before it, those conducted by both this group and other groups around the world. Funding and support for research in behavioral biology and psychology has been shrinking for some time now and a result like this should remind all of us, including those determining funding priorities, why we need to fund basic research. Important findings, such as this one, that could have critical implications for such universally supported endeavours as species preservation and conservation do not materialize solely from work directed at solving a particular problem in an applied fashion. Instead, findings such as this are the result of a solid, fundamental understanding of biology and psychology that cuts across traditional area boundaries, and are then combined by innovative, creative-minded researchers to reach beyond the initial results. In this way, the current paper, and all of the principles that led directly or indirectly to this leap forward in our understanding, should be held up as an example of how we rarely know what intellectual and practical treasures curiositydriven, fundamental research can provide if given the opportunity and resources needed to flourish.

\section{References}

Dabelsteen, T. (2005). Public, private or anonymous? Facilitating and countering eavesdropping. In P. K. McGregor (Ed.), Animal Communication Networks (pp. 38-62). Cambridge: Cambridge University Press.

Magrath, R. D., Haff, T. M., Fallow, P. M., \& Radford, A. N. (2014). Eavesdropping on heterospecific alarm calls: From mechanisms to consequences. Biological Reviews of the Cambridge Philosophical Society, 90, 560-586.

Magrath, R. D., Haff, T. M., McLachlan, J. R., \& Igic, B. (2015). Wild birds learn to eavesdrop on heterospecific alarm calls. Current Biology, 25(15), 2047-2050. doi:10.1016/j.cub.2015.06.028 